\title{
Analysis of harmonics using wavelet technique
}

\author{
Thangaraj. K ${ }^{1}$, Subramaniam.N.P ${ }^{2}$, Narmada. $\mathbf{R}^{3}$, Oma Mageswari. $\mathbf{M}^{4}$ \\ ${ }_{1,3} \mathrm{EEE}, \mathrm{SMVEC}$, India \\ ${ }^{2} \mathrm{EEE}, \mathrm{PEC}$, India \\ ${ }^{4} \mathrm{ICE}, \mathrm{SMVEC}$, India
}

\begin{tabular}{|c|c|}
\hline Article Info & ABSTRACT \\
\hline Article history: & This paper develops an approach based on wavelet technique for the \\
\hline Received Aug 11, 2018 & $\begin{array}{l}\text { estimation of harmonic presents in power system signals. The proposed } \\
\text { technique divides the power system signals into different frequency }\end{array}$ \\
\hline Revised Nov 20, 2018 & sub-bands corresponding to the odd harmonic components of the signal. \\
\hline Accepted Dec 11, 2018 & $\begin{array}{l}\text { The algorithm helps to determine both the time and frequency information } \\
\text { from the harmonic frequency bands. The comparative study will be done }\end{array}$ \\
\hline Keywords: & $\begin{array}{l}\text { With the input and the results attained from the wavelet transform (WT) for } \\
\text { different conditions and Simulation results are given. }\end{array}$ \\
\hline
\end{tabular}

Electric power quality

Harmonic distortion

Multiresolution analysis

Signal and noise

Wavelets All rights reserved.

\section{Corresponding Author:}

Thangaraj. K,

EEE, SMVEC,

Puducherry, India.

Email: thangaraj.electres@gmail.com

\section{INTRODUCTION}

Nowadays importance of harmonic studies plays a major role in Power system networks. The power quality disturbance can be caused variation in electrical power service, such as harmonics, voltage oscillations, quick disruptions and transients which results in faulty operation or failure of concern equipment. The harmonic presents in power system distorts the voltage and current signal which in turn creates various problems. Conventionally, for harmonic analysis, the discrete Fourier transform (DFT) is suggested which provides frequency information of the signal, but it will not give time information required [1]. Therefore, DFT is not a suitable for non-stationary signal.

Power quality can be improved by introducing Wavelet technique for harmonic studies to overcome the disadvantages in the conventional methods. Wavelets are a set of functions which can be used effectively to represent natural, highly transient phenomena that result from a dilation and shift of the original waveform. To analyze non-stationary signals, the Wavelet Technique is an efficient signal processing tool and also it has wide variety of applications [2].

In power quality analysis using wavelet technique, the signal will be compared to wavelet function, and a set of coefficients which is obtained gives information of correlation of wavelet function with the signal. Wavelet Transform (WT) provides good time resolution and poor frequency resolution at high frequencies and good frequency resolution and poor time resolution at low frequencies. It is useful specifically when the signal has high frequency components for short durations and low frequency components for long durations. Finally, this paper compares the performance of the results obtained using proposed wavelet transform (WT) for various conditions. 


\section{WAVELETS}

Wavelets are oscillating waveforms of short duration which amplitude decays quickly to zero at both ends. In WT, the wavelet is dilated and shifted to vary the frequency of oscillation and time location. These dilating and shifting mechanisms are very important to analyze non-stationary signals compared to conventional methods such as discrete Fourier transform (DFT) and short time Fourier transform (STFT). Wavelet technique analyses the signal at different frequencies with different resolutions. Wavelets have important properties suitable for analysis of non-stationary waveforms.

The filtering process shown in Figure 1 is the method used in most of the discrete wavelet transforms (DWT) and the first component to multiresolution analysis is vector spaces [3]. Then for every vector space, there will be higher resolution vector space till you get to the required signal. Also, every vector space comprises all vector spaces with lower resolution. The basis of each of these vector spaces is the scale function for the wavelet and signifies the detailed version of the high-frequency components of the signal and the approximation version of the low-frequency components and similarly the reconstruction process of wavelet transform which is shown in Figure 2.

The low pass filtering, A eliminates the high frequency information and high pass filtering, D eliminates low frequency information but scale remains same. During the process of subsampling, the scale will get affected. Due to filtering operations, the Resolution which is related to the quantity of information in the signal also gets affected.

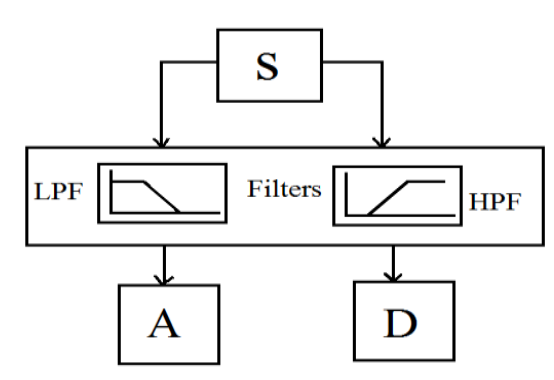

Figure 1. Filtering process

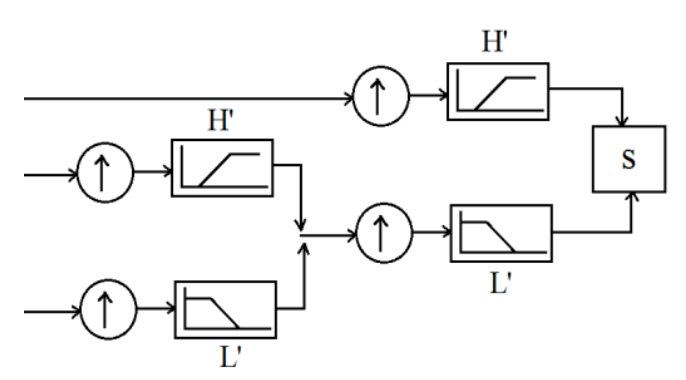

Figure 2. Wavelet reconstruction

The use of Half band low pass filter removes half of the frequencies and because of that half of the information regarding signal will be loosed. Therefore, once the operation of filter is completed then the resolution is halved [4]. However, after filtering process the subsampling operation will not affect the resolution, because anyway it removes half of the samples in the signals without losing information. The authors (in [5]) propose a method to compensate the defective response of the filters used in the wavelettransform filter banks. The new improved approach Wavelet Transform (WT) was implemented to overcome the disadvantages of conventional methods. In the WT, the details are further decomposed to produce new coefficients, this way enabling a frequency decomposition of the input signal to be obtained.

\section{PROPOSED ALGORITHM}

The algorithm proposed in this paper is wavelet transform (WT) which is compatible with the frequency bands of the different harmonic groups and uses the Daubechies 20 as the wavelet function and the filter bank with three levels of decomposition shown in Figure 3. The sampling frequency selected is $1.6 \mathrm{kHz}$ with fundamental frequency of $50 \mathrm{~Hz}$. The decomposition process can be iterated, so that one signal is broken down into many lower-resolution components and higher-resolution components respectively as shown in Figure 3 and the output frequency bands of wavelet transform shown in Figure 4. The output of the filter bank is divided into frequency bands (coefficients of $\mathrm{d} 1$ to $\mathrm{d} 4$ ) which offers information about harmonic groups presents in the input signal [6]-[7]. The flowchart for the process of wavelet transforms which is shown in Figure 5.

Each coefficient of wavelet transform measures the correlation between the signal and the basis function. If tit is large coefficients denote good correlation; on the other hand small coefficients denote poor correlation. After analyzing the presents of harmonics in the frequency sub-bands, the suitable mitigation method will be applied to those sub-bands without affecting the coefficients which contains original signal [10]. So according to the threshold value set, the wavelet technique eliminates the lower magnitude 
coefficients retains only the significant coefficients. After altering the coefficients, the decomposed components will be assembled back to get the original signal without loss of information.

The filter used in reconstruction process plays major role in attaining original signal without loss of information [6]. The reconstructed details and approximations are true constituents of the original signal. The RMS magnitude of the signals cab be calculated by using the square root of the mean square of the wavelet coefficients. During downsampling process of the signal components there is chance of a distortion called aliasing. It can be eliminated by carefully selecting filters for the decomposition and reconstruction process.

Actually, it is very much essential to get maximum flat passband characteristics and good frequency separation. According to [9], wavelet functions with a large number of coefficients will have lesser distortion when compared to lower coefficients. The Daubechies wavelet is a good processing tool for power-quality monitoring in the power system [8]. In order to measure higher range of harmonic orders (greater than 15th order), the sampling frequency and level of the decomposition will be increased in Figure 5 according to the harmonic conditions [7].

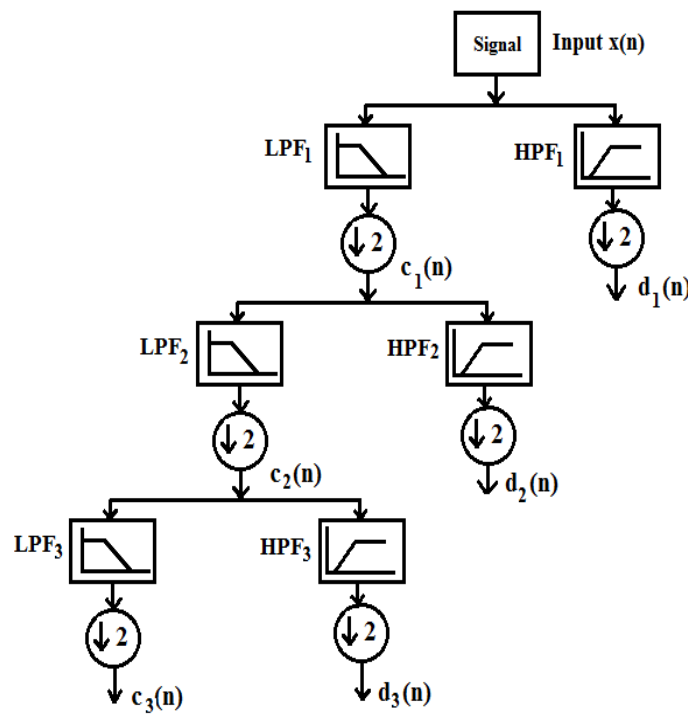

Figure 3. Three level wavelet decomposition tree

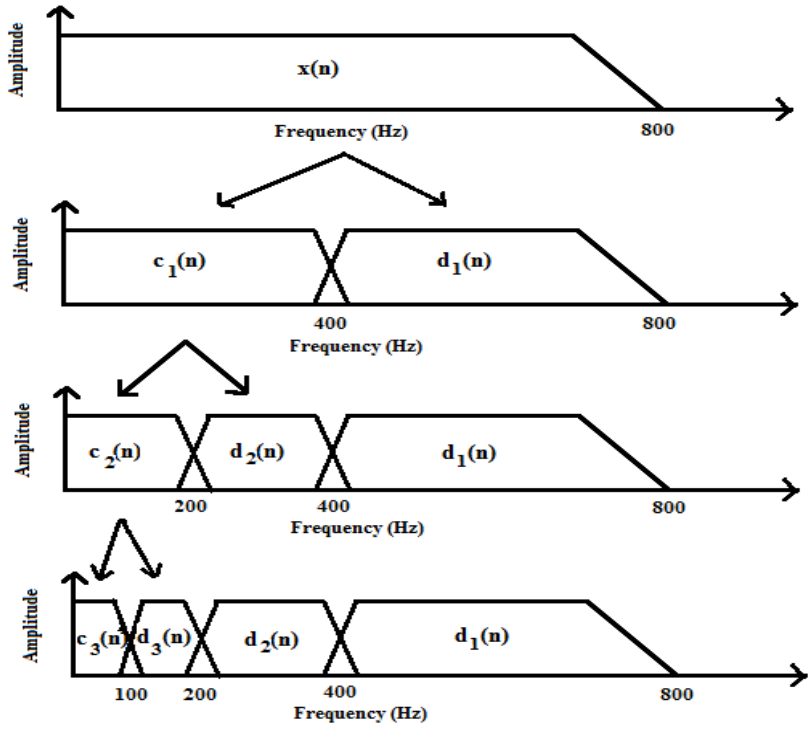

Figure 4. Output frequency bands of wavelet decomposition

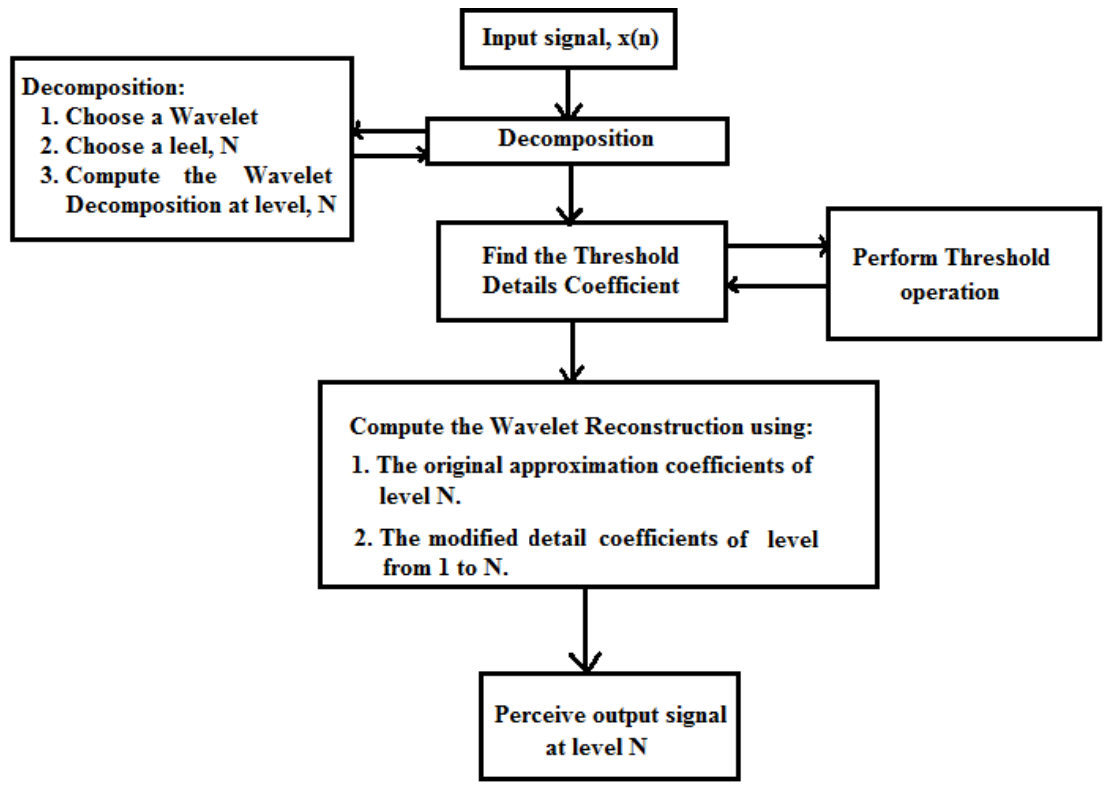

Figure 5. Flowchart for WT 


\section{SIMULATION RESULTS}

The Wavelet Transform (WT) technique for analyzing the harmonics was implemented by using the software package of MATLAB. In this section, a comparative analysis will be done with the input and the results obtained from the wavelet transform (WT) for different measuring conditions.

\subsection{Test signal 1}

Consider the input signal shown in Figure 6 which contains seventh harmonic component at every time instant in fundamental component signal of $50 \mathrm{~Hz}$. By using the analyzing information from decomposed signals, the mitigation techniques Consider the input signal shown in Figure 6 which contains seventh harmonic component at every time instant in fundamental component signal of $50 \mathrm{~Hz}$. By using the analyzing information from decomposed signals, the mitigation techniques will be applied. If mitigation techniques applied using that information, then the output signal shown in Figure 6 is obtained with error of $0.5342 \%$ which is shown in Table 1 . The location of disturbance starting and ending time are shown in Table 2 with percentage deviation and from the information, the amplitude of the given disturbance signal are observed.
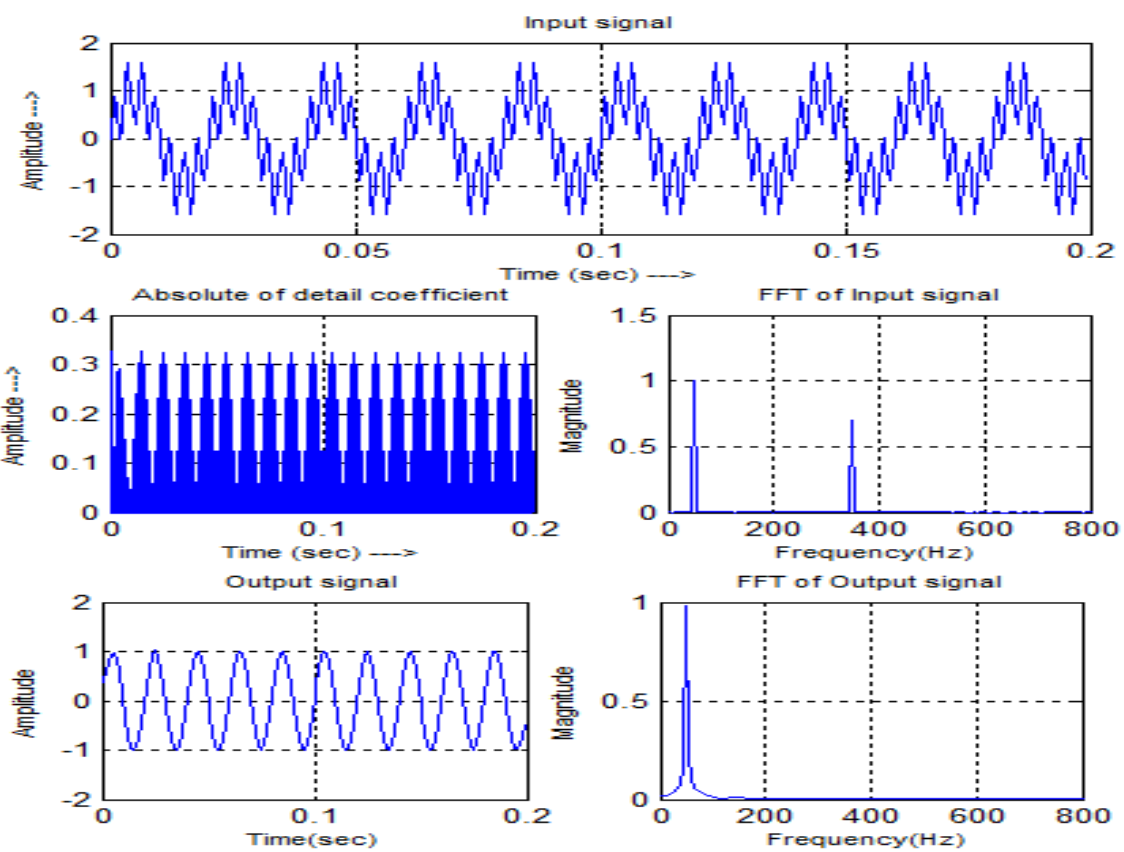

Figure 6. Harmonics at every time instant

Table 1. Error Comparison for Different Harmonic Conditions

\begin{tabular}{ccccc}
\hline \multicolumn{2}{c}{ RMS value of reference input=0.7071 } & RMS value \\
Test signals & $\begin{array}{c}\text { RMS value } \\
\text { of output }\end{array}$ & $\begin{array}{c}\text { Error (\%) } \\
\text { Comp. time } \\
(\mathrm{sec})\end{array}$ \\
\hline Harmonics at every time instant & 0.8631 & 0.7033 & 0.5342 & 0.7563 \\
Harmonics at every time instant with different frequencies & 1.0149 & 0.7150 & 1.1202 & 0.7748 \\
Harmonics at specified time & 0.8064 & 0.7009 & 0.8785 & 0.7658 \\
Harmonics at specified time with different frequencies & 0.8872 & 0.7011 & 0.8456 & 0.8760 \\
\hline
\end{tabular}

Table 2. Time and Amplitude of Disturbance Signal for Different Harmonic Conditions

\begin{tabular}{|c|c|c|c|c|c|c|c|c|}
\hline \multirow[b]{2}{*}{ Test signals } & \multicolumn{3}{|c|}{ Disturbance time $(\mathrm{sec})$} & \multicolumn{5}{|c|}{ Amplitude } \\
\hline & $\begin{array}{l}\text { Theo. Dist. } \\
\text { Time (sec) }\end{array}$ & $\begin{array}{l}\text { Act. Detected } \\
(\mathrm{sec})\end{array}$ & $\begin{array}{c}\text { Avg. } \\
\text { Deviation (\%) }\end{array}$ & Theo & litude & $\begin{array}{r}\text { Act. D } \\
\text { va }\end{array}$ & $\begin{array}{l}\text { ected } \\
e^{2}\end{array}$ & $\begin{array}{c}\text { Avg. } \\
\text { deviation }(\%)\end{array}$ \\
\hline $\begin{array}{l}\text { Harmonics at every time } \\
\text { instant }\end{array}$ & $\begin{array}{c}\mathrm{t} 1=0 \\
\mathrm{t} 2=0.2\end{array}$ & $\begin{array}{c}\mathrm{t} 1=0 \\
\mathrm{t} 2=0.2\end{array}$ & 0 & & & 0 & & 0 \\
\hline $\begin{array}{l}\text { Harmonics at specified } \\
\text { time }\end{array}$ & $\begin{array}{l}\mathrm{t} 1=0.075 \\
\mathrm{t} 2=0.15\end{array}$ & $\begin{array}{l}\mathrm{t} 1=0.0762 \\
\mathrm{t} 2=0.1506\end{array}$ & 1.00 & & & 0.9 & & 1.16 \\
\hline $\begin{array}{l}\text { Harmonics at specified } \\
\text { time with different } \\
\text { frequencies }\end{array}$ & $\begin{array}{c}\mathrm{t} 1=0.075 \\
\mathrm{t} 2=0.1 \\
62\end{array}$ & $\begin{array}{l}\mathrm{t} 1=0.0737 \\
\mathrm{t} 2=0.1631\end{array}$ & 1.02 & 0.7 & 0.9 & 0.705 & 0.89 & 0.91 \\
\hline
\end{tabular}




\subsection{Test signal 2}

The input signal which shown in Figure 7 which contains third and eleventh harmonic component at every time instant in fundamental component signal of $50 \mathrm{~Hz}$. The location of disturbance starting and ending time are shown in Table 2 with percentage deviation and from the information, the amplitude of the given disturbance signal are observed. If mitigation techniques applied using that information analyzed from decomposed signals, then the output signal shown in Figure 7 is obtained with error of $1.1202 \%$ shown in Table 1 from the figure, it is found that the wavelet transform gives the required information about the disturbance signal.
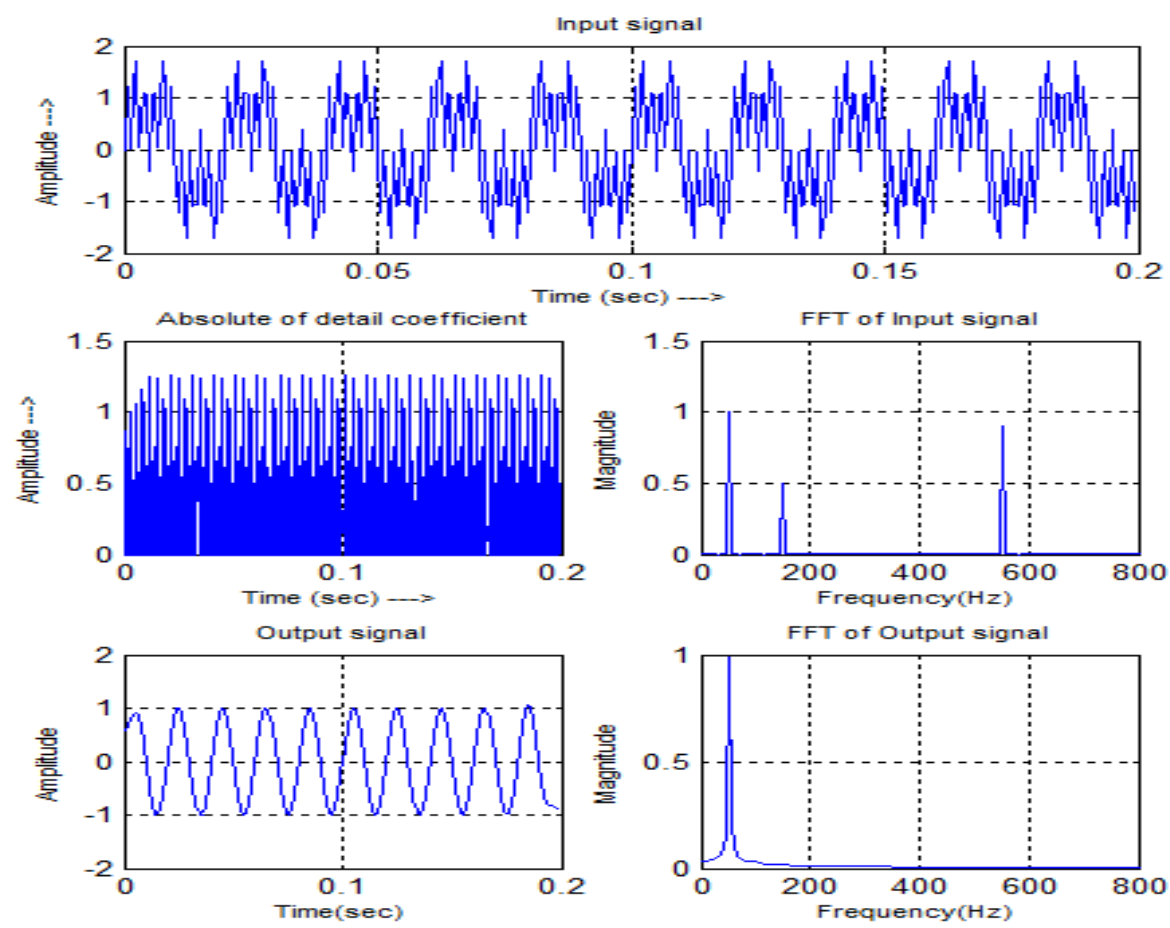

Figure 7. Harmonics at every time instant with different frequencies

\subsection{Test signal 3}

Consider the input signal shown in Figure 8 which contains third harmonic component at specific time instant in fundamental component signal of $50 \mathrm{~Hz}$. By using the analyzing information from decomposed signals, the mitigation techniques will be applied. If mitigation techniques applied using that information, then the output signal shown in Figure 8 is obtained with error of $0.8785 \%$ which is shown in Table 1. The location of disturbance starting and ending time are shown in Table 2 with percentage deviation and from the information, the amplitude of the given disturbance signal are observed.

\subsection{Test signal 4}

The input signal which shown in Figure 9 which contains third and seventh harmonic component at specific time instant in fundamental component signal of $50 \mathrm{~Hz}$. The location of disturbance starting and ending time are shown in Table 2 with percentage deviation and from the information, the amplitude of the given disturbance signal are observed. If mitigation techniques applied using that information analyzed from decomposed signals, then the output signal shown in Figure 9 is obtained with error of $0.8456 \%$ shown in Table 1. From the Figure, it is found that the wavelet transform gives the required information about the disturbance signal. The signal information errors are compared using above tabular column for different measuring conditions. From the results obtained it is found that wavelet transform has better performance for analysis of power quality disturbance. 

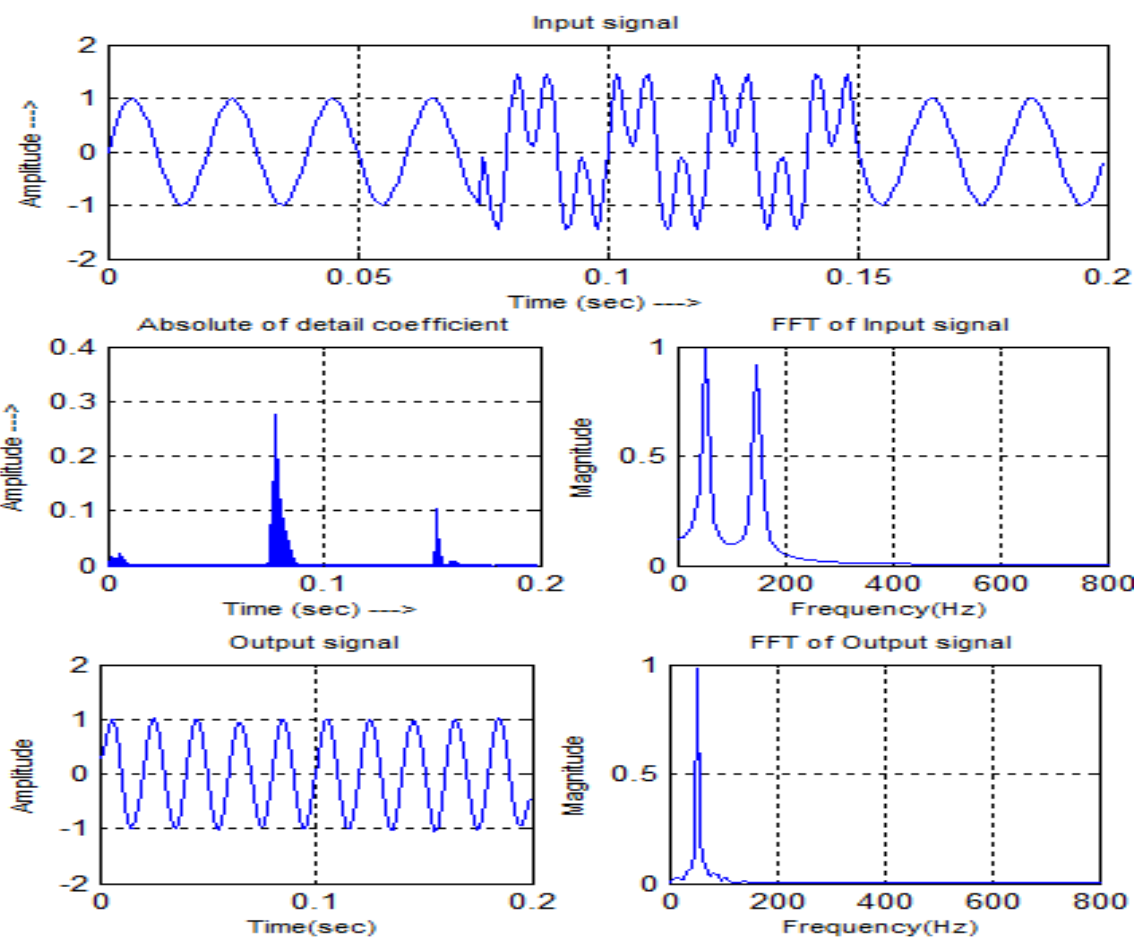

Figure 8. Harmonics at specified time
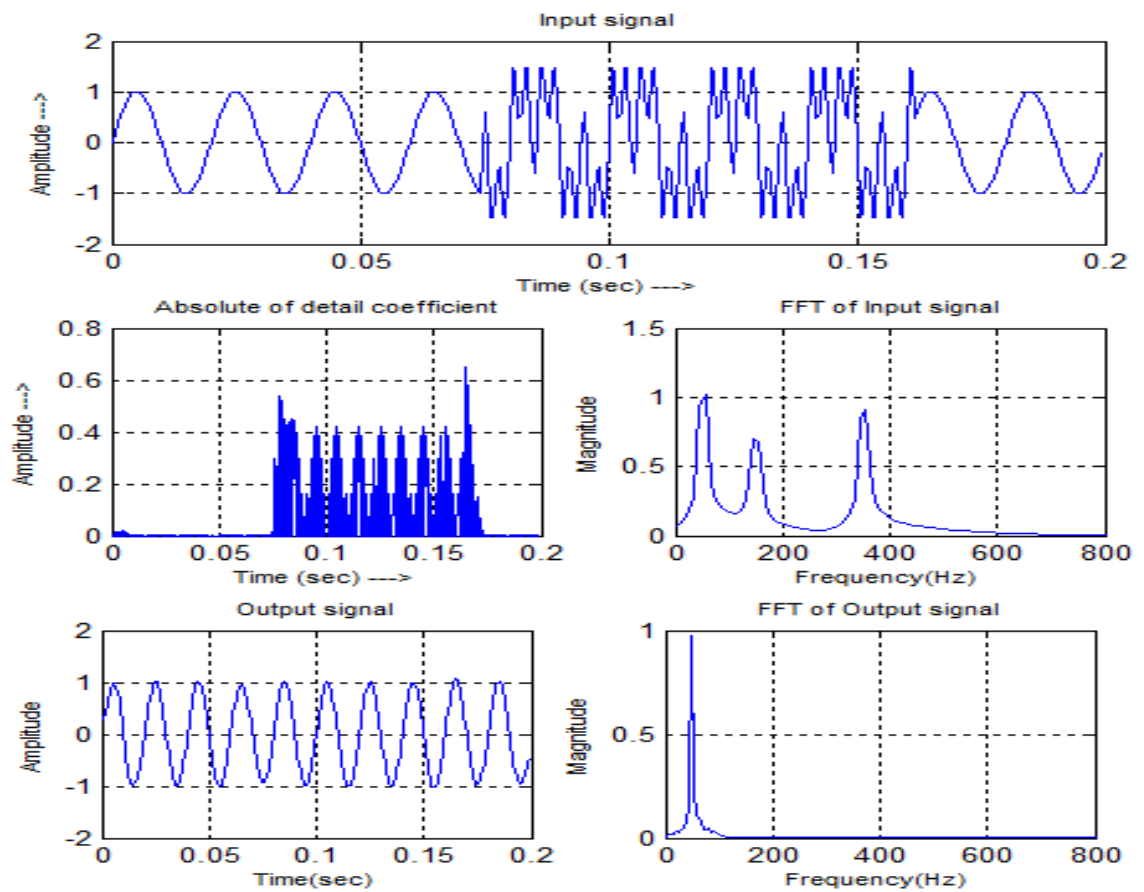

Figure 9. Harmonics at specified time with different frequencies

\section{CONCLUSION}

This paper has presented a new method of wavelet technique based algorithm for the analysis of harmonics using db20 wavelet function. Several case-studies, analyzed with different types of disturbances in electrical power system, have shown the suitability of the method. The performance of the proposed method has been compared with the input signal by calculating RMS value of the signal for different conditions and showing the wavelet technique analysis as an alternative processing tool for the harmonic estimation. 


\section{REFERENCES}

[1] Hsiung Cheng Lin, "Inter-Harmonic Identification Using Group-Harmonic Weighting Approach Based on the FFT," IEEE Transactions on Power Electronics, vol. 23, No. 3, May 2008.

[2] QU Wei, JIA Xin, PEI Shibing, WU Jie, "Non-stationary Signal Noise Suppression Based on Wavelet Analysis," Congress on Image and Signal Processing., vol. 4, May 2008.

[3] Surya Santoso, Edward J. Powers, W. Mack Grady and Peter Hofmann, "Power Quality assessment via Wavelet Transform Analysis," IEEE Transaction on Power Delivery., vol. 11,No. 2, April 1996.

[4] Kit Po wong and Van Long Pham, "Analysing Power System waveforms using Wavelet Transform Approach," Proceedings of the $5^{\text {th }}$ International Conf. on Advances in Power System Control, oper. and Management., vol. 2, Oct. 2000.

[5] V. L. Pham and K. P. Wong, "Antidistortion method for wavelet transform filter banks and nonstationary power system waveform harmonic analysis," Proc. Inst. Electr. Eng.-Gener.Transm. Distrib., vol. 148, sno. 2, pp. 117-122, Mar. 2001.

[6] Tomasz Tarasiuk, "Hybrid Wavelet-Fourier Method for Harmonics and Harmonic Subgroups Measurement-Case Study," IEEE Transactions on Power Delivery, vol. 22, No. 1, Jan 2007.

[7] EnrangZheng, Zhengyan Liu, Lingkum Ma, "Study on Harmonic Detection Method Based on FFT and

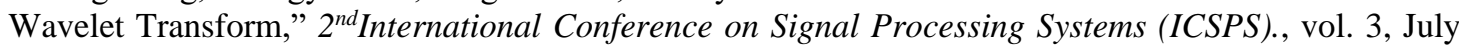
2010.

[8] Thangaraj.K, “Analysis of Harmonics using S-Transform," International conference on Emerging Trends in Engineering, Technology and Science (ICETETS), 2016.

[9] ZHU Shou-xiand LiuHui, "Simulation study of power harmonic based on Daubechies Wavelet," E-Product E-Service and E-Entertainment (ICEEE), International Conference., Nov. 2010.

[10] K.Thangaraj, "Analysis and Estimation of Harmonics using Wavelet Packet Transform," International conference on Emerging Trends in Engineering, Technology and Science (ICETETS), 2016. 